\title{
BMJ Open Effectiveness of moxibustion for allergic rhinitis: protocol for a systematic review
}

\author{
Sheng Chen, ${ }^{1}$ Shengnan Guo, ${ }^{2}$ Jun Wang, ${ }^{1}$ Eunhae Ha, ${ }^{1}$ Federico Marmori, ${ }^{3,4}$ \\ Yanping Wang, ${ }^{5}$ Jiping Zhao ${ }^{1}$
}

To cite: Chen S, Guo S, Wang J, et al. Effectiveness of moxibustion for allergic rhinitis: protocol for a systematic review. BMJ Open 2015;5:e006570. doi:10.1136/ bmjopen-2014-006570

- Prepublication history for this paper is available online. To view these files please visit the journal online (http://dx.doi.org/10.1136/ bmjopen-2014-006570).

SC and SG contributed equally to this work and should be considered co-first authors.

Received 9 September 2014 Revised 28 March 2015 Accepted 9 April 2015
CrossMark

For numbered affiliations see end of article.

Correspondence to Professor Jiping Zhao; z.jp7883@sina.com

\section{ABSTRACT}

Introduction: The objective of this review is to systematically evaluate the effectiveness of moxibustion for treating allergic rhinitis (AR).

Methods and analysis: The following databases will be searched from their inception to January 2015: MEDLINE, EMBASE, CINAHL, AMED, Web of Science, Ovid Healthstar, PubMed, SciELO, the China National Knowledge Infrastructure Database, the Chongqing VIP Chinese Science and Technology Periodical Database, the Chinese Biomedical Database, the Wanfang Database, the Japanese Medical Research Database, and Korean Medical Databases. The methodological quality will be assessed using the Cochrane risk of bias tool. The primary outcome is total nasal symptoms, which may be evaluated by any appropriate scores or other forms of measurement. Secondary outcome measures will include quality of life, total non-nasal symptoms, use of daily medication and laboratory indicators.

Ethics and dissemination: Because this study will not involve patients, institutional review body permission is not required. The systematic review will be published in a peer-reviewed journal. The review will also be disseminated electronically and in print to help guide healthcare practice and policy.

Trial registration number: PROSPERO CRD42014013275.

\section{INTRODUCTION}

Allergic rhinitis (AR) is a common disorder which often coexists with asthma in the same patient. ${ }^{1}$ The incidence of AR has risen dramatically in recent years and now affects an estimated $10-20 \%$ of the global population. ${ }^{2}$ $\mathrm{AR}$ is directly responsible for exacerbations of other inflammatory airway diseases in addition to asthma, such as rhinosinusitis and chronic otitis media, and feelings of depression and anxiety. ${ }^{3}$ Compared with several decades ago, AR now presents with higher seizure frequency and greater severity, affecting quality of life. ${ }^{4-6}$ It has been reported that AR causes severe sleep disorders leading to sleepiness interfering with daytime activities and impaired normal functioning as reflected in lower levels of productivity at school and at work. ${ }^{7}$ AR was traditionally classified as seasonal AR, perennial AR (PAR) and occupational $\mathrm{AR},{ }^{89}$ depending on the time and type of exposure and symptoms. In 2001, the Allergic Rhinitis and its Impact on Asthma (ARIA) guidelines recommended the use of the terms 'intermittent' and 'persistent'. ${ }^{10} \mathrm{AR}$ is classified as mild or moderate-severe according to the affect on quality of life, which includes sleep, work and study. Despite the development of new medications, side effects cannot be completely eliminated. ${ }^{2}$ In addition, it has been shown that AR patients often have significant medication nonadherence $^{11}$ and do not receive appropriate treatment for the most troublesome symptoms. ${ }^{12} 13$ Meanwhile, complementary and alternative medicine for treating AR is becoming more popular. ${ }^{14} 15$ A previous systematic review has shown that acupuncture to treat $\mathrm{AR}$ is effective and safe and may have some advantages over routine medical treatment. ${ }^{16}$

Acupuncture is an important component of Traditional Chinese Medicine (TCM) owing to its frequent use for the treatment of pain and other conditions. Moxibustion also plays an important role in the prevention and treatment of numerous diseases and often accompanies acupuncture treatment. ${ }^{17}$ Moxibustion is a natural therapy suitable for some chronic and severe diseases and works by stimulating acupuncture points with thermal energy from ignited moxa. Research has suggested that moxibustion exerts its effects by regulating the body's immune function via immunoglobulin, ${ }^{18}$ cytokines ${ }^{19-23}$ and the immune organs (thoracic duct and spleen). ${ }^{24}{ }^{25} \mathrm{~A}$ recent study showed that moxibustion can decrease IL- 4 levels, relieve the $\mathrm{IgE}$ inflammatory reaction, and enhance the IFN- $\gamma / \mathrm{IL}-4$ ratio in asthmatic rats. ${ }^{26}$ Published studies on moxibustion for the treatment of AR describe diverse clinical applications, with most types of moxibustion for AR demonstrating positive effects. ${ }^{27}$ However, no critically designed systematic review to evaluate the effectiveness of 
moxibustion for AR has been carried out so far. We will conduct a systematic review of moxibustion for AR to summarise any reliable evidence for clinical physicians and to help AR patients seek more treatment options. In this article, we present a protocol for this systematic review.

\section{OBJECTIVES}

The objective of this review is to systematically evaluate the effectiveness of moxibustion for treating AR.

\section{METHODS AND ANALYSES}

Study registration

Our protocol for this systematic review has been registered on PROSPERO (registration number: CRD42014013275).$^{28}$ Our review reporting will follow the Preferred Reporting Items for Systematic Reviews and Meta-Analyses (PRISMA) statement guidelines. ${ }^{29}$

\section{Study design}

Types of studies

Only randomised controlled trials (RCTs) will be included.

\section{Types of participants}

Patients with physician-diagnosed AR (repetitive sneezing, watery rhinorrhoea, nasal congestion, an itchy nose and throat, and itchy and watery eyes) will be included in this review; there will be no sex, age, source or course restrictions.

\section{Types of interventions}

Studies that involved any form of moxibustion (eg, direct moxibustion, indirect moxibustion, heat-sensitive moxibustion, moxa burner moxibustion, warm needling, crude drug moxibustion or natural moxibustion) as the sole treatment or as a part of a combination therapy with another intervention (eg, conventional drugs) will be included. Studies in which moxibustion is not used as a major therapy will be excluded.

\section{Types of outcome measures \\ Primary outcomes}

The primary outcome is total nasal symptoms, which may be evaluated by any appropriate scores or other forms of measurement, such as the Total Nasal Symptom Score (TNSS), ${ }^{30} 31$ recorded from validated daily or weekly diaries or visual analogue scales.

\section{Secondary outcomes}

Secondary outcome measures will include:

1. Quality of life: evaluated by any general and/or disease-specific scales, such as the Rhinoconjunctivitis Quality of Life Questionnaire (RQLQ) ${ }^{32}$

2. Global non-nasal symptoms: recorded in validated daily or weekly diaries and any scores from any validated visual analogue scales, such as the Total Non-Nasal Symptom Score (TNNSS).$^{31}$
3. Use of conventional medication: assessed by an instrument such as the Medication Quantification Scale (Version III ${ }^{33}$ to record the administration frequency and quantity of AR relief medication.

4. Laboratory indicators: such as eosinophil count and/ or serum IgE levels or any other validated index.

\section{Security outcomes}

1. Adverse events: any unexpected events occurring during the studies will be recorded on an adverse event report form.

2. Laboratory indicators: any relevant examination, such as white blood cell, alanine aminotransferase, aspartate aminotransferase, blood urea nitrogen or serum creatinine, will be recorded in order to monitor the safety of studies.

\section{Search methods}

\section{Checking registries and databases}

The Cochrane Central Register of Controlled Trials (CENTRAL), the International Clinical Trials Registry Platform (ICTRP), the International Prospective Register of Systematic Reviews (PROSPERO), ClinicalTrials.gov, and the University Hospital Medical Information Network-Clinical Trials Registry (UMIN-CTR) will be searched up to January 2015 for reviews on moxibustion and AR. The Cochrane Library and the Cochrane Database of Systematic Reviews (CDSR), as well as the Educational Resources and Information Center (ERIC), BEME, DARE, NICE and the Campbell Library of Systematic Reviews databases will be searched for published or submitted systematic reviews or protocols.

\section{Searching databases}

The following databases will be searched from their inception to January 2015: MEDLINE, EMBASE, CINAHL, AMED, Web of Science, Ovid Healthstar, PubMed, SciELO, China National Knowledge Infrastructure Database (CNKI), the Chongqing VIP Chinese Science and Technology Periodical Database (VIP), the Chinese Biomedical Database (CBM), the Wanfang Database, the Japanese Medical Research Database and Korean Medical Databases. Terms to be used for searching include (moxibustion $\mathrm{OR}$ moxa $\mathrm{OR}$ warm needling $\mathrm{OR}$ vesiculation $\mathrm{OR}$ blister) AND (allergy OR allergies OR [allergic AND rhinitis] OR hay fever OR pollinosis). Specific search strategies (eg, for PubMed) are as follows:

\#1. (( ( moxibustion [Title/Abstract]) OR moxa [Title/ Abstract]) OR warm needling [Title/Abstract]) OR vesiculation [Title/Abstract]) OR blister [Title/Abstract]

\#2. ((( (allergic rhinitis [Title/Abstract]) OR allergy [Title/Abstract]) OR allergies [Title/Abstract]) OR hay fever [Title/Abstract]) OR pollinosis [Title/Abstract]

\#3. (( (random [Text Word] OR randomized [Text Word]) OR control [Text Word]) OR controlled [Text Word]) OR trial [Text Word] AND "humans" [MeSH Terms]

\section{\#4. \#1AND\#2AND\#3}


We will use similar search strategies for other electronic databases.

\section{Study selection}

Studies that involve any form of moxibustion (eg, direct moxibustion, indirect moxibustion, heat-sensitive moxibustion, moxa burner moxibustion, warm needling moxibustion, crude herb moxibustion, natural moxibustion or medicinal vesiculation) as the sole treatment or as a part of a combination therapy with another intervention (eg, conventional drugs) will be selected. However, studies in which moxibustion is not used as a major therapy will be excluded. Studies in which one form of moxibustion is compared with another form will be excluded. Studies will be restricted to human subjects, but there are no restrictions on inclusion criteria or language. Studies will be collected and screened by four review authors working in pairs, and will be checked by $\mathrm{EH}$ who is Korean and JZ who is fluent in Japanese. When disagreements arise, JZ will make a final decision based on discussion. The entire process of selecting studies is shown in the PRISMA flow diagram (figure 1).

\section{Data extraction}

Data will be independently extracted by two authors, and will include the names of article authors, year of publication, country, sample size, randomisation method, blinding, participants, intervention, duration, outcomes, observation points, results, follow-up and adverse effects. Information will be recorded in a data extraction table. For literature published in abstract or dissertation form with important details regarding methods and results

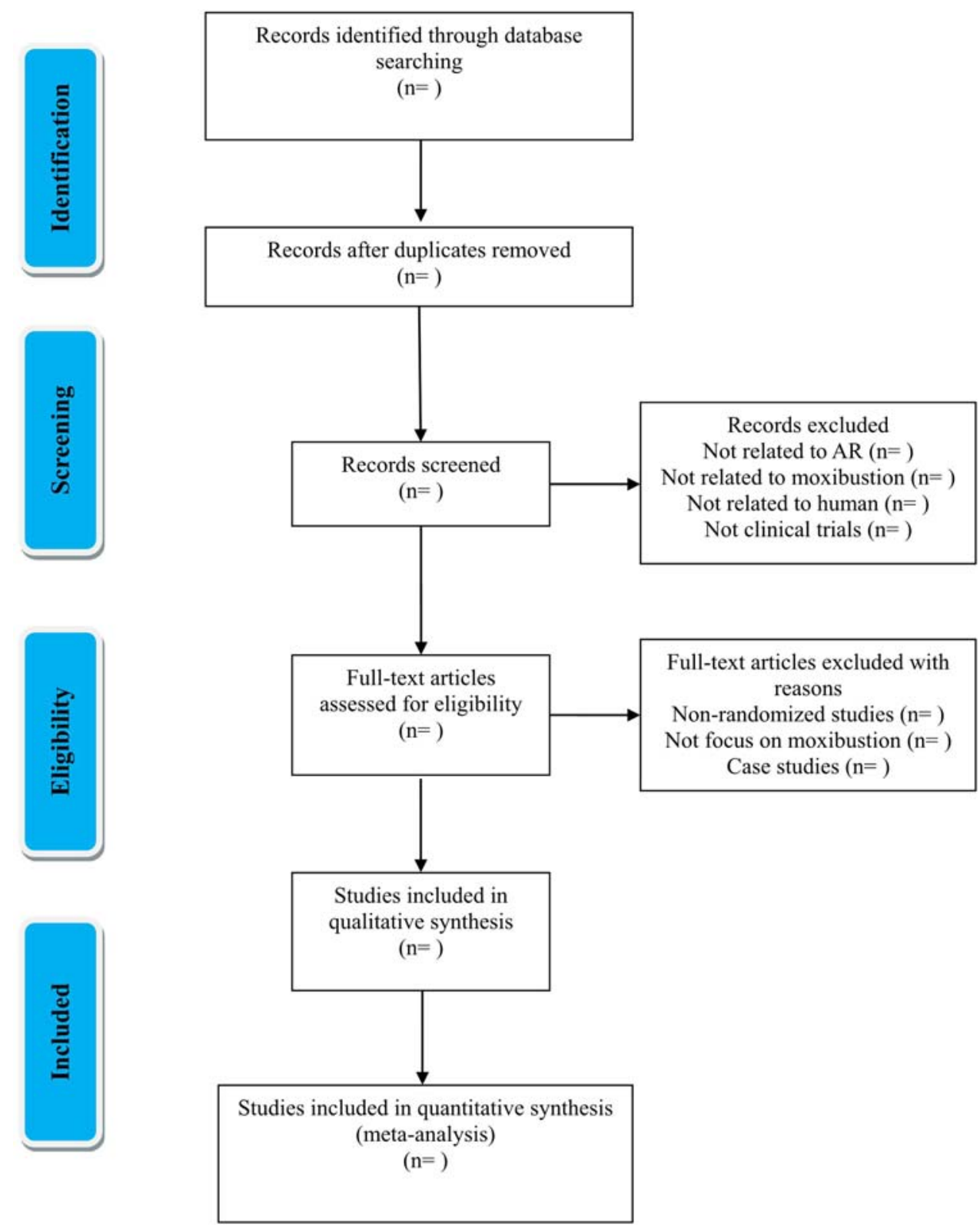

Figure 1 Study selection flow diagram. AR, allergic rhinitis. 
missing, we will try to retrieve information from the authors.

\section{Quality assessment}

The Cochrane risk of bias tool, ${ }^{34}$ which is recommended by the Cochrane Reviewer's Handbook 5.0.24, will be used to assess the quality of the included studies. Two review authors will independently evaluate the quality of selected articles, and will assess selection bias (random sequence generation or allocation concealment), performance bias and detection bias (blinding), attrition bias (incomplete outcome data), reporting bias (selective outcome reporting) and other biases..$^{35}$ If necessary, we will contact the corresponding author to clarify issues. Any disagreements will be resolved through discussion or consultation with a third reviewer.

\section{Data synthesis}

Analysis of data collected will depend on its characteristics (participants, interventions, comparisons, and outcomes or methodology). If there is excessive data heterogeneity, we will use qualitative analysis to summarise the study. When meta-analysis is feasible, quantitative analysis will be used to process data, and each form of moxibustion intervention will be assessed separately.

We will conduct all statistical analyses with Cochrane Collaboration's software (Review Manager (Rev Man) V.5.3) for Windows. Before data meta-analysis, heterogeneity will be tested with a standard $\chi^{2}$ test. ${ }^{36}$ For studies with high heterogeneity $\left(\mathrm{p}>0.10, \mathrm{I}^{2} \leq 50 \%\right)$, the fixed-effect model will be used. For dichotomous and continuous data, ${ }^{37}$ we will use the relative risk (RR) and mean difference with 95\% CIs for measurement, respectively. For studies with low heterogeneity $(p \leq 0.10)$, subgroup or sensitivity analysis will be performed. Finally, the total effect will be tested by using the $\mathrm{Z}$ score with significance set at $\mathrm{p} \leq 0.05$. Funnel plots will be used to detect the possibility of publication bias. ${ }^{35}$

\section{Subgroup analysis}

If the necessary data are available, subgroup analyses will be carried out according to different factors as follows:

1. Control interventions (eg, sham/placebo moxibustion, no treatment, other TCM treatment or non-TCM treatment)

2. Type of moxibustion (eg, direct moxibustion, indirect moxibustion, heat-sensitive moxibustion, moxa burner moxibustion, warm needling moxibustion, crude drug moxibustion or natural moxibustion)

3. Treatment frequency (eg, less than three times per week versus more than three times per week)

4. Classification of AR (eg, intermittent, persistent, seasonal or PAR).

\section{DISCUSSION}

To our knowledge, most of the current studies describing complementary and alternative medicine involve acupuncture treatment. There are no systematic reviews of moxibustion treatment for AR. This is the first protocol for a systematic review designed to assess the effectiveness of moxibustion for AR patients. Although we will collect the relevant literature without language restrictions through an extensive and unbiased search of various databases, we cannot be certain that our search will include all relevant RCTs. Additionally, we may have difficulty in retrieving raw data from published sources. The publications or reports we select to search is another possible major cause of bias. Previous research concerning acupuncture for AR showed that the total numbers of articles and sample sizes were limited. ${ }^{39}$ However, we believe the results of this study could provide evidence on the effectiveness of moxibustion in treating AR, which will benefit patients and practitioners.

\section{Author affiliations}

${ }^{1}$ Department of Acupuncture and Moxibustion, Dongzhimen Hospital Affiliated to Beijing University of Chinese Medicine, Beijing, China

${ }^{2}$ Institute of Acupuncture and Moxibustion, China Academy of Chinese Medical Sciences, Beijing, China

${ }^{3}$ European Foundation of TCM, Alicante, Spain

${ }^{4}$ Acupuncture Department, Medimar International Hospital, Alicante, Spain

${ }^{5}$ School of Acupuncture, Moxibustion and Tuina of Beijing University of Chinese Medicine, Beijing, China

Contributors SC and SG conceived the study, developed the criteria and searched the literature, performed data analysis and wrote the protocol. JW, $\mathrm{EH}$ and $\mathrm{JZ}$ assisted in searching the literature and extracting data. FM and YW wrote the introduction of this protocol. JZ advised on the protocol design and revised the manuscript. All authors read and approved the final manuscript.

Funding Capital research on the clinical characteristic application projects is funded by Beijing Municipal Science \& Technology Commission (D101100050010022).

Competing interests None declared.

Provenance and peer review Not commissioned; externally peer reviewed.

Open Access This is an Open Access article distributed in accordance with the Creative Commons Attribution Non Commercial (CC BY-NC 4.0) license, which permits others to distribute, remix, adapt, build upon this work noncommercially, and license their derivative works on different terms, provided the original work is properly cited and the use is non-commercial. See: http:// creativecommons.org/licenses/by-nc/4.0/

\section{REFERENCES}

1. Greiner AN, Hellings PW, Rotiroti G, et al. Allergic rhinitis. Lancet 2012;378:2112-22.

2. Brozek JL, Bousquet J, Baena-Cagnani $\mathrm{CE}$, et al. Allergic Rhinitis and its Impact on Asthma (ARIA) guidelines: 2010 revision. J Allergy Clin Immunol 2010;126:466-76.

3. Hadley JA, Derebery J, Marple BF. Comorbidities and allergic rhinitis: not just a runny nose. J Fam Pract 2012;61:S11-15.

4. Xu PR, Li M. Recent advances in allergic rhinitis: diagnosis and treatment. J Clin Pediatr 2007;25:4-7.

5. Mösges R, Klimek L. Today's allergic rhinitis patients are different: new factors that may play a role. Allergy 2007;62:969-75.

6. Deng YQ, Tao ZZ. The relationship between allergic diseases and psychological factors. Int J Otorhinolaryngol Head Neck Surg 2009;33:81-3.

7. Craig TJ, Sherkat A, Safaee S. Congestion and sleep impairment in allergic rhinitis. Curr Allergy Asthma Rep 2010;10:113-21.

8. Van Cauwenberge P, Bachert C, Passalacqua G, et al. Consensus statement on the treatment of allergic rhinitis. Allergy 2000;55:116-34.

9. Dykewicz MS. 7. Rhinitis and sinusitis. J Allergy Clin Immunol 2003;111:S520-9. 
10. Bousquet $\mathrm{J}$, Van Cauwenberge $P$, Khaltaev N. Allergic rhinitis and its impact on asthma. J Allergy Clin Immunol 2001;108: S147-334.

11. Valovirta E, Ryan D. Patient adherence to allergic rhinitis treatment: results from patient surveys. Medscape J Med Chem 2008;10:247.

12. Meltzer EO. Allergic rhinitis: the impact of discordant perspectives of patient and physician on treatment decisions. Clin Ther 2007;29:1428-40.

13. Stauder A, Kovács M. Anxiety symptoms in allergic patients: identification and risk factors. Psychosom Med 2003;65:816-23.

14. Passalacqua G, Bousquet PJ, Carlsen $\mathrm{KH}$, et al. ARIA update: - Systematic review of complementary and alternative medicine for rhinitis and asthma. J Allergy Clin Immunol 2006;117:1054-62.

15. Schafer T, Riehle A, Wichmann HE, et al. Alternative medicine in allergies: prevalence, patterns of use, and costs. Allergy 2002;57:694-700.

16. Xiao L, Li B, Du YH, et al. Systematic evaluation of the randomized controlled trials about acupuncture and moxibustion treatment of allergic rhinitis. Zhongguo Zhen Jiu 2009;29:512-16.

17. Liangyue D, Yijun $\mathrm{G}$, Shuhui $\mathrm{H}$, et al. Chinese acupuncture and moxibustion. Tradit Med Asia 2001:75.

18. Wu HG, Wang JH. The effect and immunological mechanism of cake separated moxibustion in treatment of irritable bowel syndrome. Zhongguo Zhen Jiu 1996;16:43-5.

19. Xie S, Li LH, Lv MZ. Experimental study on the effect of moxibustion at Zusanli (ST36) and Guanyuan (CV4) on the immune function of aging mice. J Guiyang Coll Tradit Chin Med 2003;25:44-6.

20. Su L, Li L, Yang JS, et al. Modern research on the effect of moxibustion on body function. Chin J Inf Tradit Chin Med2010;17:101-3.

21. Tang ZL, Song XG. Experimental study on anti-inflammatory and immunological mechanism of moxibustion. J Anhui Univ Chin Med 2003;22:31-5.

22. Tang ZL, Song XG, Chen QZ, et al. Experimental study on anti-inflammatory and immunological mechanism and the effect on neurotransmitter of moxibustion. Chin J Basic Med Tradit Chin Med 2005;6:53-5.

23. Yang ZX, Zhao YX. Study on strengthening mice immunity and antineoplastic function of moxibustion. Chin Arch Tradit Chin Med 2002;20:94-5.

24. Tang ZL, Song XG, Hou ZM, et al. Experimental study on anti-inflammatory and immunization of moxibustion [J]. Acupuncture Res 1997;22:168-70.

25. Tang ZL, Song XG. Experimental study on anti-aging by moxibustion at Shenshu (BL23). J Anhui Univ Chin Med 1999;18:53-5.
26. Zhang W, Chen MR, Xiong J. Effects of different quantity of moxibustion at 'Dazhui' (GV14) on cellular immunity in asthma rats. Acupunct Res 2012;37:202-5.

27. Liu Q, Yang J, Zhao BX. Overview of different moxibustion methods treating allergic rhinitis. World Chin Med 2014;9:923-7.

28. PROSPERO. PROSPERO International prospective register of systematic reviews. http://www.crd.york.ac.uk/PROSPERO/display record.asp?ID=CRD42014013275

29. Moher D, Liberati A, Tetzlaff J, et al. Preferred reporting items for systematic reviews and meta-analyses: the PRISMA statement. Ann Intern Med 2009;151:264-9.

30. Food and Drug Administration. Guidance for industry: allergic rhinitis: clinical development programs for drug products (draft guidelines). Available at: http://www.fda.gov/cder/guidance/2718dft htm (accessed 7 July 2005).

31. Spector SL, Nicklas RA, Chapman JA, et al. Symptom severity assessment of allergic rhinitis: part 1. Ann Allergy Asthma Immunol 2003;91:105-14.

32. Juniper EF, Thompson AK, Ferrie PJ, et al. Validation of the standardized version of the Rhinoconjunctivitis Quality of Life Questionnaire. J Allergy Clin Immunol 1999;104:364-9.

33. Harden RN, Weinland SR, Remble TA, et al. Medication quantification scale version III: update in medication classes and revised detriment weights by survey of American Pain Society Physicians. J Pain 2005;6:364-71.

34. Higgins JPT, Altman DG, Gøtzsche PC, et al. The Cochrane Collaboration's tool for assessing risk of bias in randomised trials. BMJ 2011;343.

35. Higgins JPT, Altman DG, Sterne JAC. Chapter 8: assessing risk of bias in included studies. In: Higgins JPT, Green S, eds. Cochrane Handbook for Systematic Reviews of Interventions Version 510 (updated March 2011). The Cochrane collaboration, 2011. http:// www.cochrane-handbook.org

36. Higgins J, Thompson SG. Quantifying heterogeneity in a meta-analysis. Stat Med 2002;21:1539-58.

37. Akl EA, Johnston BC, Alonso-Coello P, et al. Addressing dichotomous data for participants excluded from trial analysis: a guide for systematic reviewers. PLOS ONE 2013;8:e57132.

38. Ebrahim S, Akl EA, Mustafa RA, et al. Addressing continuous data for participants excluded from trial analysis: a guide for systematic reviewers. J Clin Epidemiol 2013;66:1014-21.e1.

39. Lee MS, Pittler MH, Shin BC, et al. Acupuncture for allergic rhinitis: a systematic review. Ann Allergy Asthma Immunol 2009;102:269-79. 